\author{
Anna Grochowska-Reiter \\ Università "Adam Mickiewicz" di Poznań \\ Daniel Stapek ${ }^{* *}$ \\ Università di Breslavia
}

\title{
DISCORDANZE TEORICO-PRATICHE NELLA PRESENTAZIONE DI ALCUNE NORME GRAMMATICALI AL LIVELLO A1/A2 NELLE GRAMMATICHE DI ITALIANO LS/L2
}

\begin{abstract}
Con il presente lavoro intendiamo esaminare come vengono presentati, nelle grammatiche d'italiano L2, vari argomenti relativi alla fascia di competenza A del Quadro comune europeo di riferimento (QCER), tra i quali: a) pronomi personali soggetto (lui, lei vs egli, ella) e la forma di cortesia al plurale (voi vs loro), b) particella ci con i verbi essere (c'è, ci sono) e avere (ce l'ho ecc.), c) flessione verbale: verbi irregolari al presente indicativo, imperativo irregolare (forme tronche), d) semantica del verbo: venire vs andare e potere, sapere vs riuscire, ed e) aggettivi possessivi: l'uso dell'articolo determinativo davanti ai singenionimi. Dalle nostre analisi, effettuate su quindici grammatiche italiane per stranieri che fanno un esplicito riferimento al QCER, pubblicate da note case editrici, si evincono alcuni punti deboli quanto all'insegnamento della grammatica, quali l'omissione totale di un argomento, una certa selettività (un dato problema non viene presentato nella sua completezza), nonché varie discordanze teorico-pratiche nella presentazione delle norme grammaticali agli apprendenti stranieri.

Parole chiave: italiano lingua non materna, grammatica italiana, grammatica italiana per stranieri, insegnamento della grammatica, QCER, didattica della lingua italiana $L S$.
\end{abstract}

\footnotetext{
*anna_g@amu.edu.pl

** daniel.slapek@uwr.edu.pl
} 


\section{INTRODUZIONE}

Diversi dubbi ed errori grammaticali sorti nella prima fase dell'apprendimento di una lingua straniera, se non sufficientemente spiegati, commentati ed esercitati, sono difficili da sradicare anche ai livelli di competenza più alti. Per questo motivo, l'introduzione del materiale linguistico che andrebbe studiato al livello base risulta particolarmente importante.

Nelle pagine che seguono vogliamo commentare alcuni argomenti grammaticali, il cui insegnamento si colloca grosso modo nella fascia A (QCER) - secondo i sillabi di riferimento per la lingua italiana non materna -, tra i quali ${ }^{1}$ :

- $\quad$ i pronomi personali soggetto, inseriti in tutti i sillabi al livello $\mathrm{Al}^{2}$; ci concentreremo sulle forme della $3^{\text {a }}$ persona singolare $l u i$, lei vs egli, ella nonché sulla forma di cortesia plurale voi vs loro, indicata expressis verbis solo nel Sillabo di italiano L2: "pronomi allocutivi: tu, lei per il singolare, voi per il plurale" (Lo Duca 2006: 183);

- la particella pronominale $c i$ con i verbi essere e avere; il valore locativo di $c i$ viene indicato al livello A1, invece la struttura ce l'ho è del tutto trascurata, presente solo nel sillabo di Lo Duca (2006) al livello B1; tuttavia, riteniamo che - per motivi pragmatici - sia più lecito introdurla già nella fascia $\mathrm{A}$;

- la flessione verbale: verbi irregolari al presente indicativo (collocati, in linea di massima, tra i livelli A1 e A2, a seconda della loro frequenza d'uso) e le forme tronche dell'imperativo irregolare (A1/A2);

- alcuni aspetti della semantica del verbo: differenze semantiche e funzionali dei verbi venire vs andare (argomento assente in tutti i sillabi presi in esame) e potere, sapere vs riuscire (i sillabi si concentrano soprattutto sui verbi modali propriamente detti, potere, dovere, volere, sempre nella fascia A; sapere viene indicato solo in Arcangeli et al. 2014: 47; Accademia Italiana di Lingua 2015: 4);

- l'uso dell'articolo determinativo davanti ai nomi di parentela preceduti da un aggettivo possessivo (solo il Sillabo per i cinque gradi... di Minciarelli e Comodi (2005) colloca gli aggettivi possessivi a un livello più alto: A2/B1).

${ }^{1}$ Tabella 1 indica gli argomenti grammaticali presi in esame insieme ai loro livelli di competenza linguistica secondo alcuni sillabi di riferimento per la lingua italiana (per un'analisi più dettagliata dei sillabi/linee guida qui citati si veda anche Słapek 2019).

${ }^{2}$ Fanno eccezione i sillabi di Minciarelli \& Comodi (2005) insieme ai quaderni delle specifiche di Plida che inseriscono i pronomi soggetto al livello A2; tuttavia, i due titoli non propongono alcun inventario grammaticale per il livello A1. 


\begin{tabular}{|c|c|c|c|c|c|c|c|c|}
\hline & $\begin{array}{c}\text { Acca- } \\
\text { demia } \\
\text { Italiana } \\
\text { di Lingua } \\
(2015)\end{array}$ & $\begin{array}{c}\text { Arcan- } \\
\text { geli } \\
\text { et al. } \\
(2014)\end{array}$ & $\begin{array}{c}\text { Barni } \\
\text { et al. } \\
(2009)\end{array}$ & $\begin{array}{c}\text { Benucci } \\
(2007)\end{array}$ & $\begin{array}{c}\text { Cardillo } \\
\text { \& } \\
\text { Vecchio } \\
2015\end{array}$ & $\begin{array}{c}\text { Lo } \\
\text { Duca } \\
(2006)\end{array}$ & $\begin{array}{c}\text { Min- } \\
\text { ciarelli, } \\
\& \text { Co- } \\
\text { modi } \\
(2005)\end{array}$ & $\begin{array}{c}\text { Spi- } \\
\text { nelli \& } \\
\text { Parizzi } \\
(2010)\end{array}$ \\
\hline $\begin{array}{c}\text { pronomi } \\
\text { soggetto }\end{array}$ & A1 & A1 & A1 & A1 & A2 & A1 & A2 & A1 \\
\hline $\begin{array}{c}\text { forma di } \\
\text { cortesia } \\
\text { plurale }\end{array}$ & - & - & - & - & - & A1 & - & - \\
\hline $\begin{array}{c}\text { c'è̀... } \\
\text { ce 1'ho... }\end{array}$ & - & - & - & - & - & - & - & B1 \\
\hline $\begin{array}{c}\text { andare } v s \\
\text { venire }\end{array}$ & - & - & - & - & - & - & - & - \\
\hline $\begin{array}{c}\text { potere, } \\
\text { sapere, } \\
\text { riuscire }\end{array}$ & A1/A2 & A1 & A1 & A1 & A2 & A1/A2 & - & A2 \\
\hline $\begin{array}{c}\text { aggettivi } \\
\text { possessivi }\end{array}$ & A1 & A1/A2 & A1 & A1/A2 & A2 & A1/A2 & A2 & A1 \\
\hline $\begin{array}{c}\text { verbi } \\
\text { irregolari } \\
\text { al presente }\end{array}$ & A1 & A1/A2 & A2 & A1/A2 & A2 & A1 & A2/B1 & A1/A2 \\
\hline imperativo & A2 & A1/A2 & A1 & A2 & - & A1 & - & A2 \\
\hline
\end{tabular}

Tabella 1. Collocazione di alcuni argomenti grammaticali nei livelli QCER secondo i sillabi di riferimento per l'italiano L2/LS

Tutti gli argomenti grammaticali di cui sopra vengono trattati al livello base dell'insegnamento, anche se in varia misura e con diversa attenzione: alcuni usi particolari di una data categoria possono, ad esempio, essere distribuiti tra due o addirittura tre livelli di competenza del QCER (p. es. i verbi irregolari introdotti a seconda della loro frequenza tra i livelli A1 e B1; si veda Tabella 1). L'obiettivo principale delle nostre analisi è, quindi, - come suggerisce lo stesso titolo - di dimostrare alcune discordanze nella presentazione del suddetto materiale linguistico nelle grammatiche italiane per stranieri e di commentarne le eventuali lacune ${ }^{3}$.

${ }^{3} \mathrm{Ci}$ rendiamo conto che alcuni problemi di cui parleremo vanno oltre il livello A1. Crediamo tuttavia che i materiali didattici per gli stranieri debbano presentare la grammatica in maniera esaustiva. Benché tutti i titoli presi in esame superino la soglia A1, alcuni commenti grammaticali ivi esposti risultano incompleti. 


\section{GRAMMATICA NELLE GRAMMATICHE ITALIANE PER STRANIERI}

Nell'analisi entrano le grammatiche italiane per stranieri pubblicate a partire dal 2001 da note case editrici specializzate, tra l'altro, nella didattica d'italiano LS/L2, i cui autori si riferiscono esplicitamente al QCER e i cui volumi coprono i livelli A1 e A2. Rimangono, quindi, fuori interesse le grammatiche che partono da livelli più avanzati nonché numerosi eserciziari di grammatica privi di regole grammaticali come tali.

Sono stati sottoposti all'analisi i seguenti volumi (i titoli sono preceduti dalle loro sigle che, per comodità, verranno usate nel corso del testo):

- CI: Comunicare in italiano (Chiuchiù e Chiuchiù 2015);

- GAt: Grammatica attiva (Landriani 2012);

- GB: Grammatica di base (Esposito e Errico 2007);

- GBI: Grammatica di base dell'italiano (Petri et al. 2015);

- GdU: Grammatica d'uso della lingua italiana (Celi e La Cifra 2011);

- GiC: Grammatica in contesto (Gatti e Peyronel 2006);

- G.it: Gramm.it (Iacovoni et al. 2009);

- GP: Grammatica pratica della lingua italiana (Mezzadri 2016);

- GS: Grammatica della lingua italiana Per Stranieri, vol. 1 (Tartaglione e Benincasa 2015);

- GT: Una grammatica italiana per tutti 1, vol. 1 (Latino e Muscolino 2014);

- IE: Italiano essenziale (Mezzadri 2003);

- LSR: La lingua italiana e le sue regole (Debetto 2016);

- NGP: Nuova grammatica pratica della lingua italiana (Nocchi 2011);

- UD: L'utile e il dilettevole 1 (Ercolino e Pellegrino 2011);

- VG: Via della grammatica (Ricci 2011).

\subsection{L'uso dei pronomi personali soggetto (il caso di egli ed ella)}

Purtroppo, non tutti i volumi dedicano un capitolo a parte ai pronomi personali soggetto, ma l'argomento viene comunque trattato, soprattutto nell' esposizione dell'indicativo presente o, ancora di più, con i verbi essere e avere. Tra le soluzioni che ci hanno stupito si annovera l'uso dei pronomi egli, ella per la terza persona singolare (per essere precisi, sempre insieme a lui e lei). Nella maggior parte dei casi - fortunatamente - troviamo un'apposita annotazione, come p. es. "nell'italiano moderno, egli, ella, essa si trovano raramente" (GP, p. 16; similmente in IE, p. 83; LRS, p. 151); GB è più precisa a questo riguardo: "l'uso di questi pronomi è raro e limitato alla lingua scritta e burocratica" (p. 23; infatti, il primo commento potrebbe suggerire l'uso di egli/ella nella lingua parlata, anche se poco frequente). 
Crediamo che sia poco opportuno citare i detti pronomi nelle tabelle di coniugazione senza alcuna nota di approfondimento, come in GAT (p. 23), dove nella coniugazione del verbo essere troviamo "(egli) lui / (ella) lei / lei" (nel capitolo "pronomi personali soggetto" dello stesso titolo appaiono solo lui e lei, per cui risulta poco coerente; ivi, p. 142). Ė ancora meno fortunata la dicitura "egli [ed ella] è la forma giusta per la grammatica, lui [e lei] è la forma d'uso" (CI, p. 2). Senza entrare nel dibattito sull'opposizione tra la linguistica e la grammatica tradizionale (a questo proposito si veda p. es. Colombo \& Graffi 2017), troviamo alquanto scoraggiante insegnare agli studenti principianti che la grammatica e l'uso della lingua sono due fenomeni separati. Tenendo in mente lo standard linguistico e nel contempo l'uso effettivo e la lingua comune (l'italiano dell'uso medio descritto già da Francesco Sabatini nel 1985 e l'italiano neostandard da Gaetano Berruto nel 1987), ci sembra ragionevole che ai livelli iniziali vengano favoriti i pronomi lui, lei, loro. Ciò, ovviamente, non esclude la possibilità di spiegazione dei pronomi egli, essa, esso purché sia chiara, coerente ed esaustiva e ne chiarisca doverosamente le differenze funzionali e stilistiche.

\subsection{La forma di cortesia al plurale}

Sono ancora meno numerosi i titoli che parlano dei pronomi personali soggetto nel registro formale (tre titoli dedicano un capitolo a parte alle forme di cortesia: GP, p. 16; VG, p. 47; GdU, p. 6), specie per quanto riguarda la loro forma del plurale. In tal modo, troveremo i pronomi voi ("al plurale usiamo sempre voi: sia per la forma di cortesia [...] sia quando la situazione è informale", GP, p. 16; similmente in GBI, p. 87; VG, p. 47; IE, p. 84) e Loro, il quale pronome a) viene indicato come "raro": "è raro trovare il soggetto loro per la forma di cortesia al plurale" (GP, p. 16; IE, p. $84)$; b) viene definito come particolarmente formale: "la forma di cortesia al plurale Loro è sentita come molto formale ed è per lo più usata sostituita da voi" (GB, p. 23; similmente in GiC p. 27; VG, p. 47), "in situazioni molto formali o quando c'è una differenza considerevole d'età tra gli interlocutori, si usa il pronome Loro (per il plurale)" (GBI, p. 87); c) è indicato come in disuso: "la forma di cortesia Loro +3 a persona plurale non si usa più; si usa Voi $+2^{\mathrm{a}}$ persona plurale" (GdU, p. 6). Inoltre, GAt indica per il plurale solo loro: "Lei Loro (formale) / Per comunicare con persone non conosciute: Prego, Signori, entrino anche Loro! [...] Al bar: Che cosa prendono (Loro) da bere?" (p. 23) ${ }^{4}$. A nostro avviso, gli autori delle grammatiche sono troppo

${ }^{4}$ Tutti gli autori concordano sull'uso del pronome Lei (al singolare), ma anche a questo proposito troviamo alcuni interessanti commenti: "il pronome Lei nella forma di cortesia nella lingua scritta ha la lettera maiuscola. Ma questa regola non si usa quasi più" 
sbrigativi e spesso cadono nella superficialità e imprecisione. Soprattutto nei casi come questo, in cui la dimensione sociale della struttura grammaticale gioca il ruolo fondamentale, dovrebbero evitare le spiegazioni poco precise o intuitive, che lasciano troppo spazio all'interpretazione, o addurre esempi poco attendibili. Considerando che i volumi in questione si rivolgono a studentesse e studenti di tutto il mondo, provenienti da culture più disparate, le questioni riguardanti la cortesia linguistica non possono essere date per scontate ed esigono commenti esaustivi, corredati di esempi credibili, inseriti nelle situazioni reali affinché l'apprendente non solo impari la struttura in sé, ma anche capisca in quale contesto usarla.

\subsection{L'uso del verbo esserci}

Il verbo esserci, con le sue forme c'è e ci sono, è, insieme ad essere, uno dei verbi più frequenti in assoluto ${ }^{5}$ nonché uno dei primi in cui lo studente d'italiano LS si imbatte. All'infuori del suo significato principale (esistere, essere presente, trovarsi in un luogo), esserci ne possiede altri, altrettanto cruciali nella prima fase di apprendimento. Tuttavia, nonostante l'argomento sia stato riscontrato in tutte le grammatiche sottoposte all'analisi, la sua esposizione è lontana dall'essere soddisfacente ed esaustiva. Il principale significato del verbo viene esposto nella maggior parte dei volumi (tranne che in IE, CI e GiC, in cui, si noti, non si trova alcuna spiegazione in merito e del verbo esserci vengono presentate solamente le forme); 1'altro significato (avere luogo, svolgersi) è decisamente meno discusso (GB, p. 13), similmente alle forme c'è / ci sono associate ai sostantivi indicanti i fenomeni atmosferici (c'è il sole, c'è il vento, ci sono le nuvole, c'è la nebbia; solo in GdU, p. 8). Rara risulta anche la riflessione sulle essenziali differenze sintattico-semantiche tra i verbi essere ed esserci: 1'ordine basico del primo (SVO) e l'ordine marcato del secondo (VSO), nonché le rispettive sfumature di significato. Le segnalazioni in merito si trovano in GS (p. 25), GB (p. 13), mentre nel caso di GdU (p. 8) e GAt (p. 26) la questione dell'ordine marcato viene sollevata, ma senza ulteriori confronti con il verbo essere. Infine, è vano cercare nelle grammatiche altri significati del verbo esserci (p. es. comprendere, arrivarci), espressioni comuni o strutture che lo contengono (p. es. C'è nessuno?, strutture come c'è da infinito, c'è / ci sono + nome + per qn). Gli unici, modesti riscontri si rinvengono in

(GdU, p. 6). A nostro avviso, invece, pur essendo sempre meno frequente, la maiuscola rimane ancora presente e per questo il suo uso andrebbe ancora raccomandato.

${ }^{5}$ Quanto alla frequenza, il Corpus e Lessico di Frequenza dell'Italiano Scritto (CoLFIS; si veda Bertinetto et al. 2005) colloca il verbo essere al primo posto ed esserci al settimo. 
LSR, con l'espressione che cosa c'è (p. 58), in GB, con C'è Carla? - Si, c'è / No, non c'è (p. 13) e in CI con Chi c'è / Chi c'è stato / Chi sarà?, Non c'è nessuno (p. 74).

L'uso di c'è e ci sono non deve annoverarsi tra gli argomenti spigolosi della grammatica italiana. Purtroppo, considerando la poca attenzione dedicatagli dalle grammatiche italiane (e, di conseguenza, dagli eserciziari e, quindi, probabilmente anche dagli insegnanti stessi), l'assimilazione di questa struttura si dimostra difficoltosa. Infatti, gli errori di comunicazione che ne risultano continuano a essere presenti nell'interlingua degli apprendenti di livelli B e perfino $\mathrm{C}$.

\subsection{L'uso della struttura ce l'ho / non ce l'ho}

Attenzione ancora minore viene concessa all'uso della particella ci nelle risposte alle domande polari contenenti il verbo avere: Hai un cane? - Si, ce l'ho / No, non ce l'ho. L'argomento è stato affrontato in otto grammatiche del corpus (NGP, p. 140; GT, p. 26; GB, pp. 13 e 150; IE, p. 91; CI, p. 14; GdU, p. 82; GAt, p. 147; GiC, p. 137), in cinque (GT, GB, CI, GdU, GAt) sono stati proposti opportuni esercizi di rinforzo ${ }^{6}$. Gli autori appaiono titubanti su dove affrontare l'argomento in questione, collocandolo o quando espongono il presente indicativo del verbo avere (GT, p. 26; GB, p. 13; CI, p. 14), o nei capitoli dedicati alla particella ci (NGP, p. 140; IE, p. 91; e ripresa in GB, p. 150), o ancora, insieme ai pronomi diretti (GdU, p. 82; GAt, p. 147; GiC, p. 137).

Lo scarso interesse rivolto a questo elemento e i dubbi su dove inserirlo difficilmente si spiegano, vista non solo la sua semplicità e brevità, ma anche utilità comunicativa. Considerando quanto esposto e ribadendo il fatto che si tratta di una delle prime strutture di cui lo studente d'italiano LS viene a conoscenza a livello A1, per lo più assente nelle principali lingue europee (Hai la macchina-Si, ce l'ho / No, non ce l'ho), sarebbe opportuno discuterla introducendo il verbo avere. In questo modo, l'apprendente non solo ottiene uno strumento comunicativo semplice ed efficace, ma anche familiarizza, pur non essendone cosciente, con i pronomi di complemento oggetto diretto, che conoscerà più avanti e che potrebbero essere introdotti proprio partendo da queste preconoscenze.

${ }^{6} \mathrm{Si}$ noti che IE è l'unico libro di grammatica del nostro corpus che non offre alcun esercizio di rinforzo. 


\subsection{L'uso dei verbi andare $e$ venire}

Manca nelle grammatiche d'italiano per stranieri l'argomento che mette a confronto l'uso dei verbi andare e venire. La coniugazione di entrambi viene puntualmente presentata in tutte le grammatiche sottoposte all'esame: in due (GT, p. 39; GdU, p. 18) andare e venire vengono affrontati in un capitolo/paragrafo separato. Sono rari, sbrigativi e non sempre facilmente intelligibili gli approfondimenti riguardanti il loro significato (p. es. "[usiamo venire] quando il movimento deriva da un invito o dalla necessità di raggiungere un luogo/una persona"; GT, p. 39); talvolta vi si affiancano frasi d'esempio o mini-dialoghi contenenti sia il significato di base (GT, p. 39, CI, p. 28, ) sia alcune espressioni più comuni, tra cui Come va?, va bene (GT, p. 39, GdU, p. 18), ti va ...? (GT, p. 39), andare/venire a trovare (GT, p. 39), venire da (GT, p. 39, CI, p. 28). A titolo di curiosità, in GT troviamo paragrafi dedicati alle differenze tra il verbo partire e venire, come anche l'avviso che, contrariamente al francese, in italiano il verbo andare non si usa per formare il tempo futuro (p. 39). È altamente probabile che una tale mancanza si spieghi con il fatto che le maggiori lingue europee condividono, a grandi linee, una ripartizione di funzioni semantiche simile all'italiano: aller - venir in francese, go - come in inglese, gehen/fahren - kommen in tedesco. Tuttavia, vi sono lingue come il polacco, in cui la coppia iśćljechać - przychodzić corrisponde all'uso di andare e venire solamente in parte. L'allargamento del raggio della ricerca ad altre lingue europee e un'attenta analisi dei verbi in questione e dei loro equivalenti su diversi piani semantici gioverebbe indubbiamente a sciogliere questo nodo della didattica dell'italiano.

\subsection{Differenze tra $i$ verbi potere, sapere $e$ riuscire}

I verbi modali propriamente detti (dovere, potere, volere) sono un punto immancabile nelle grammatiche italiane per stranieri. Nella maggioranza dei casi vengono affrontati in un capitolo separato (NGP, p. 38; GP, pp. 3438, 44; GT, p. 34-35; VG, p. 42; GdU, p. 42; GAt, pp. 58-59; GiC, p. 38; LSR, p. 78-79), in altri, più raramente, insieme alle forme del presente dei verbi irregolari (GBI, p. 127; GB, p. 20; IE, p. 17; CI, p. 28; UD, p. 42). Il concetto non risulta particolarmente difficoltoso, quindi la coniugazione dei verbi, qualche esempio d'uso e alcuni esercizi di rinforzo paiono essere più che sufficienti per un'adeguata acquisizione.

Invece, il problema che si pone al centro dell'attenzione nell'insegnamento dell'italiano riguarda l'uso appropriato del verbo potere, spesso confuso con sapere e ancor di più con riuscire. La quantità dilagante dei materiali on line, prodotti anche in lingue diverse dall'italiano, dedicati a 
questo argomento conferma inequivocabilmente che il problema riguarda apprendenti di diverso background linguistico ${ }^{7}$. Ciò nonostante le grammatiche moderne per stranieri stentano ancora ad aggiornare i loro contenuti: si noti che alcuni dei volumi del corpus sono ristampe e riedizioni "rivedute e aggiornate" delle grammatiche presenti sul mercato da anni, come quella di Mezzadri (2003) o le grammatiche della casa editrice Alma Edizioni; pare, tuttavia, che si tratti di un ammodernamento grafico piuttosto che contenutistico. Dall'analisi condotta risulta che il verbo potere è stato messo a confronto con sapere in otto volumi (GS, p. 39; NGP, p. 38; GT, p. 44; VG, p. 42; GAt, p. 59; GiC, p. 38; LSR, p. 78), mentre solamente in tre anche con riuscire (GS, p. 39; GT, p. 44; GAt, p. 59). Non in tutte le opere la relazione tra $i$ due o $i$ tre verbi è esplicita: ad esempio LSR dedica un capitolo ai verbi modali e il verbo sapere: nella parte teorica ripercorre i tre verbi modali, spiegandone il significato e le particolarità semantiche, invece al verbo sapere dedica una riga di testo all'inizio ("Anche il verbo sapere può reggere un infinito e assumere funzione modale: Sai nuotare?", p. 78) e due righe alla fine, segnalando che si tratta di un verbo che nei tempi composti richiede l'ausiliare avere. Anche gli esercizi di rinforzo si concentrano unicamente sui verbi modali, escludendo il verbo sapere, e simili incoerenze non sono un caso isolato ${ }^{8}$. Riassumendo, sembra indispensabile rivedere le potenziali questioni spinose riguardanti i verbi modali, soprattutto in confronto a sapere e ancor di più riuscire, senza trascurare, per quanto possibile, il contributo della grammatica contrastiva.

\subsection{Omissione dell'articolo determinativo davanti ai nomi di parentela preceduti da un possessivo}

Tra le regole più complesse - perché limitate a un gruppo molto ristretto di sostantivi all'interno di una stessa categoria semantica - troviamo l'uso (o l'omissione) dell'articolo determinativo davanti agli aggettivi possessivi seguiti dai nomi di parentela: nel caso dei singenionimi l'articolo viene omesso, eccetto alcuni casi particolari. In base a quanto è detto nelle grammatiche, possiamo elencare cinque casi in cui si richiede la presenza dell'articolo: 1) il sostantivo è riferito al plurale (p. es. GBI, p. 55: le mie sorelle); 2) il

${ }^{7}$ Si pensa, ad esempio, a numerosi episodi dei $v \log$ dedicati allo studio dell'italiano L2, tra cui p. es. Ask an Italian Teacher - Expressing Ability: Potere or Riuscire, POTERE and How to Say CAN in Italian (facilmente rintracciabili su www.youtube.com).

${ }^{8} \mathrm{GS}$ nella parte teorica discute potere, sapere, riuscire, nella parte pratica propone un unico esercizio di completamento riguardante solo i primi due verbi (p. 44); GiC si limita agli esercizi cumulativi in cui chiede di coniugare i verbi forniti tra parentesi, senza proporre esercizi mirati per i verbi modali o verbo potere e sapere, affrontati nella parte teorica (pp. 41-42). 
sostantivo è preceduto dal possessivo loro (p. es. VG, p. 62: il loro zio); 3 ) il sostantivo è determinato in qualche maniera; si parla a questo proposito di "determinanti" (BG, p. 82: il mio caro nonno), di aggettivi (GAT, p. 101: il mio fratello preferito) o complementi (UD, p. 68: il tuo zio d'America); 3 ) il nome è alterato (p. es. GS, p. 51: il mio cuginetto); 4) si tratta di forme affettive (GS, p. 51: il mio papà); 5) si tratta di nomi prefissati (CI, p. 47: la mia bisnonna).

Le grammatiche citano solitamente le prime due categorie di sostantivi (fa eccezione LRS che si dimentica del possessivo loro), ma sono molto meno attente quanto agli altri tipi dei nomi di parentela: 4 titoli non prendono in considerazione i nomi alterati (GP, IE, GiC, LSR), 5 non parlano della determinazione del sostantivo (GP, GT, IE, GDU, GiC), addirittura 6 tralasciano la questione dei nomi affettivi (GP, GT, IE, GDU, GiC, LSR). La categoria più trascurata, per così dire, è quella dei nomi prefissati, riferiti solo in due volumi (CI, p. 47; UD, p. 68). Il titolo più esaustivo - da questo punto di vista - è CI (p. 47), l'unico a considerare tutte le categorie di cui sopra.

Inoltre, l'uso dell'articolo davanti ai possessivi e nomi affettivi andrebbe considerato facoltativo, in quanto "nell'italiano familiare, specie fuori dalla Toscana, sono tuttavia ben saldi i tipi mia mamma e mio papà" (Bongi 2008), per cui "le grammatiche [tranne, purtroppo, quelle per stranieri] e, da un po' di tempo, anche i dizionari dell'uso considerano pienamente accettabili" queste strutture (si veda Treccani, s.d.).

\subsection{Verbi irregolari al presente indicativo}

La maggior parte delle grammatiche per stranieri dedica un capitolo a parte ai verbi irregolari al presente indicativo. Abbiamo, quindi, verificato quali verbi vengono esposti (coniugati per intero) e ne risulta che: 1) sono 40 i verbi considerati dagli autori (tra cui 36 possono essere classificati, secondo il dizionario della lingua italiana di De Mauro (2000), con le marche d'uso FO, quindi fondamentali, mentre gli altri 4 verbi hanno la marca $\mathrm{AU}$, di alto uso); 2) le grammatiche più complete da questo punto di vista sono9: GB (con 32 verbi), NGP (30 verbi), GDU (26 verbi), seguono VG e GiC (22 verbi); i titoli più limitati sono GS, CI, LSR (con 14 verbi); 3) 12

${ }^{9}$ I verbi irregolari sono esposti in diverse unità di un dato volume per cui, per comodità, non indichiamo qui i numeri di relative singole pagine. 
verbi si trovano in tutte le grammatiche ${ }^{10}$, invece 10 verbi sono presentati solo da un titolo ${ }^{11}$.

Sono decisamente rari i titoli che cercano di raggruppare le irregolarità: 1) IE parla dei composti di alcuni verbi (es.: contenere, comporre, ritrarre, intervenire; p. 18); 2) GdU mette in rilevo i verbi uscenti in-urre (tradurre, produrre, condurre, ridurre; p. 36); 3) GB indica un insieme di "verbi in -urre,-orre,-arre" (tradurre, proporre, estrarre; p. 17); 4) UD dice ancora che i verbi "in-gliere e in-gnere, alla prima persona singolare e alla terza persona plurale, invertono le consonanti" (p. 42), invece "i verbi che terminano in -nere, alla prima persona singolare e alla terza persona plurale, prendono la $g$ davanti alla desinenza [come rimanere, ottenere] (ibid.; UD indica inoltre i gruppi in-orre e-urre). Altri titoli presentano soltanto un elenco (più o meno limitato) di verbi irregolari.

Nei testi esaminati non mancano annotazioni poco chiare, come $\mathrm{p}$. es. "con le persone io e loro, alcuni verbi comuni hanno una $g$ che non c'è nell'infinito e nelle altre persone del presente indicativo, così come tenere, porre e venire. Ecco i più comuni: rimanere, salire, valere" (IE, p. 18; una simile indicazione non aiuta in realtà a stabilire quali verbi entrano in questa categoria) o insolite classificazioni dei verbi irregolari (tra i quali GDU annovera sostituire, starnutire, tossire, obbedire, obbligare, riempire; p. 38).

Abbiamo, inoltre, confrontato l'elenco di tutti i verbi irregolari presenti nelle grammatiche con i loro ranghi d'uso secondo il lemmario del CoLFIS, che include 5194 voci verbali in totale. I 12 verbi di cui nella nota 10 si trovano tra i primi 100 verbi del CoLFIS ${ }^{12}$; altri verbi di questo gruppo sono (tra parentesi il numero dei titoli che presenta la coniugazione di un dato verbo): sapere (14 titoli), tenere (12), rimanere (9), scegliere (7), piacere (6), morire (5), riuscire (4), ottenere (1), proporre (1), parere (1), apparire (1).

Crediamo che sia opportuno presentare nelle grammatiche un vasto ventaglio dei verbi irregolari (tra cui in primo luogo i più frequenti verbi del CoLFIS), anche se - chiaramente - alcune voci potranno essere più adeguate ai livelli più alti ${ }^{13}$. Sarebbe, invece, del tutto lecito raggruppare

${ }^{10}$ Questi sono: essere, fare, avere, potere, dire, dovere, volere, andare, stare, dare, venire, uscire.

${ }^{11}$ Tra i quali: ottenere $(\mathrm{GdU})$, proporre $(\mathrm{GB})$, produrre $(\mathrm{GiC})$, parere $(\mathrm{NGP})$, apparire (GDU), raccogliere (GB), fuggire (sic!; NGP), tacere (GDU), udire (GB), estrarre (GB).

${ }^{12}$ I primi 6 addirittura occupano i primi sei posti dell'elenco.

${ }^{13}$ In realtà il problema del presente irregolare non viene poi approfondito nei livelli B e C. 
i verbi a seconda delle loro irregolarità flessive, preferibilmente con degli appositi esercizi per ogni gruppo di verbi.

\subsection{Imperativo irregolare (tu)}

La compresenza di alternative forme flesse di un dato verbo è un fenomeno abbastanza frequente in italiano (cfr. p. es. Słapek 2016: 86). Fra tali verbi troviamo andare, dare, fare e stare nella seconda persona singolare dell'imperativo che "presenta una duplice forma: oltre alle forme in - $a$ (nella grafia in genere con apostrofo: da', sta', $f a^{\prime}, v a^{\prime}$, ma si trova anche $d a, s t a$, $f a$ e $v a$ ) si hanno anche quelle forme in -ai (analogiche sull'indicativo): dai, stai, fai, vai" (Salvi e Vanelli 2004: 96).

Tuttavia, le grammatiche italiane discordano a proposito della suddetta duplice - o in realtà triplice - natura flessiva dei quattro verbi: c'è chi, infatti, ne indica due varianti (p. es. Dardano e Trifone 1995: 336-7: va' (vai), da' (dai) ecc.), c'è chi ne registra tre, per l'appunto (p. es. Sensini 1997: 324: va (o va' o vai), dà (o da' o dai) ecc.). Variano anche le opinioni sulle forme brevi accentate: "per quel che riguarda questi quattro verbi, le uniche forme d'imperativo che non esistono, e che dunque non si devono usare, sono quelle con l'accento: stà, dà, fà, và'" (Della Valle e Patota 2015: 169; si paragoni la voce dà citata da Sensini, come sopra).

Completa il quadro il verbo dire, la cui seconda persona singolare dell'imperativo è $d i$ ' apostrofata (Sensini 1997: 338 anche in questo caso ammette l'accento: $d i$ ' o $d i$ ).

Le grammatiche per stranieri sono più attente a questo riguardo: l'unica forma citata per dire è $d i$ ', per gli altri verbi si incontrano invece: 1) una sola forma apostrofata, $v a$ ', $d a^{\prime}$ ecc. (p. es. GDU, p. 108); 2) due forme alternative, in $-a$ ' $\mathrm{o}-a i$ (è la soluzione decisamente più frequente), anche se diversamente citate: varia sia l'ordine in cui le forme sono presentate, vale a dire prima le voci apostrofate seguite dalle forme piene (p. es. GP, p. 126) o viceversa (GB, p. 205), sia il modo in cui si indica l'alternanza flessiva, cioè con una barra $\left(f a^{\prime} / f a i\right.$, p. es. GT, p. 151), una parentesi $\left(d a^{\prime}(d a i)\right.$ ecc., GAT, p. 75) o tramite congiunzione (va' oppure vai ecc.; G.it, p. 214); 3) tre forme flesse: va (vai/va'); fa (fai/fa'); sta (stai/sta'); dà (dai/da'); così citate in CI (pp. 177-8; si noti la forma accentata dà) $)^{14}$.

Abbiamo controllato varie tavole di coniugazione dei verbi italiani, quindi sussidi didattici cui si ricorre più spesso per risolvere dubbi sulla flessione verbale. Ne risulta che tutti i verbi di cui sopra registrano almeno tre forme (incluso dire): andare: $v a, v a i, v a$ ' (De Renzo, 2013, p. 27), similmente fare: fa, fai, fa' (ivi, p. 80), stare: sta, stai, sta' (ivi, p. 188), dire:

${ }^{14}$ GS inspiegabilmente non tratta il tema dell'imperativo. 
dì, di', di (Parodi, 2001, p. 413), o addirittura quattro forme di dare: dà, dai, $d a$ ' (ivi, p. 99) e $d a$ (riportato in Chiuchiù, Fazi \& Bagianti 2007: 71, che però non considera la forma accentata $d a ̀$ ).

Davanti a queste numerose scelte nella presentazione dell'imperativo dei verbi irregolari, a volte anche contrastanti, crediamo che sia opportuno citare, nei materiali dedicati a stranieri, le due forme su cui concordano tutti gli autori ( $v a$ ', vai, ecc.), eventualmente insieme alle forme meno frequenti $(v a, f a, d a, s t a)$ accompagnate però da un'apposita nota in cui si spiegano sia la vera alternanza flessiva delle forme uscenti in $-a^{\prime} \mathrm{o}-a i$ sia le forme rare, ma ancora in uso. Sarebbe opportuno, magari nella stessa nota, indirizzare gli apprendenti verso l'uso più frequente.

\section{CONCLUSIONI}

L'analisi condotta sul corpus di quindici grammatiche d'italiano destinate ad apprendenti stranieri a partire dal livello A1/A2 e senza distinzione di background linguistico ha dimostrato che in molti punti i volumi si rivelano incoerenti e insoddisfacenti. In base agli argomenti sottoposti all'esame si possono individuare tre principali tipologie di problemi:

1) Omissione totale di un argomento; tra quelli analizzati, il caso più palese riguarda la struttura ce l'ho nelle domande polari, tralasciata in sette grammatiche del corpus. Vale la pena attirare l'attenzione anche sulla scarsa riflessione dedicata all'uso dei verbi andare e venire, la cui coniugazione viene puntualmente presentata in tutte le grammatiche, ma è vano cercare una spiegazione esauriente quanto ai contesti d'uso.

2) Selettività; dall'analisi del corpus risulta che raramente un dato problema grammaticale viene esposto nella sua completezza. Parlando dei livelli bassi A1/A2, si potrebbe giustificare questa manchevolezza spiegando che si tratta della fase iniziale dell'apprendimento, in cui non necessariamente un dato argomento deve essere analizzato nella sua pienezza. Eppure, le problematiche come l'uso del verbo esserci, la forma di cortesia o la differenza nell'uso di potere e riuscire sono questioni su cui difficilmente si torna ai livelli più alti. Inoltre, i volumi sottoposti all'analisi non dichiarano di coprire unicamente i livelli A1 o A2, bensì l'insieme delle fasce A e B, arrivando talvolta perfino al C1.

3) Discordanze teorico-pratiche; come si evince dall'esame condotto, vi sono $i$ casi in cui $i$ testi grammaticali propongono spiegazioni nettamente discordanti o imprecise (cfr. le forme di cortesia voi e Loro in diversi volumi), che possono indurre in errore e mettere 
in difficoltà gli apprendenti, e talvolta perfino gli insegnanti stessi. Le discordanze si osservano inoltre nella presentazione di forme flesse dei verbi (cfr. le forme dell'imperativo di alcuni verbi di alta frequenza).

In più, i problemi grammaticali analizzati nel presente lavoro sono in gran parte quelli che creano difficoltà anche agli apprendenti dei livelli elevati. Le cause sono da cercare nella poca attenzione che vi è stata dedicata all'inizio del processo di apprendimento?

Il libro di grammatica destinato agli apprendenti stranieri deve essere un punto di riferimento sicuro, ordinato e completo, che non permetta allo studente di perdersi nei meandri grammaticali; tuttavia, anche se dovesse succedere, dovrebbe essere in grado di guidarlo alla soluzione, anche senza l'aiuto dell'insegnante. Per quanto possibile, gli autori dovrebbero riflettere sui potenziali ostacoli linguistici nell'apprendimento, dovuti alle differenze tra il sistema linguistico di partenza e quello di arrivo, proponendo un quadro grammaticale più completo possibile piuttosto che ricalcare $\mathrm{i}$ vecchi schemi, spesso manchevoli.

Oltre al principio di completezza, gli spunti per colmare le lacune presenti nelle grammatiche sono da trarre, in primo luogo, da un'approfondita e capillare osservazione degli apprendenti provenienti da diversi ambienti linguistici affinché si individuino i problemi più impellenti. Un'altra fonte preziosa da cui attingere, per individuare le mancanze e i punti deboli delle grammatiche per stranieri, sono i social media: i blog sparsi nell'universo digitale, i vlog su YouTube, la web tv, i fan page su Facebook, Instagram ed altri si (im)pongono come un nuovo punto d'incontro tra chi insegna e chi impara l'italiano (si pensa, ad esempio, a ALMA.tv, la web tv dedicata alla lingua e alla cultura italiana, ai numerosi canali YouTube gestiti dalle scuole di lingue, dalle maggiori case editrici o persone private, tra cui Oneworlditaliano, Edilingua; Sgrammaticando, Learn Italian with Lucrezia, Italiano automatico, Italy made easy, e molti altri). Sono essi, con migliaia di iscritti e follower volenterosi a migliorare il loro italiano, a costituire un'inestimabile fonte di ispirazione nonché la miniera di indizi su quello con cui gli apprendenti provenienti da tutto il mondo hanno difficoltà e su quali elementi della grammatica italiana danno loro filo da torcere.

Non ci vogliamo illudere che si possa creare una grammatica che soddisfi le esigenze di tutti e prenda in considerazione il loro background linguistico per eliminare i potenziali ostacoli, ma vogliamo credere che l'obiettivo primario di una grammatica è quello di essere completa ed esaustiva, di dare a chi apprende le spiegazioni oggettive, precise, efficaci e approfondite, conformi alle norme della lingua standard. 


\section{BIBLIOGRAFIA}

\section{Sillabi di italiano L2/LS esaminati}

Accademia Italiana di Lingua (2015). AIL presentazione ed introduzione. 6 esami di italiani, da A1 a C2, \& 2 esami di italiano commerciale per tutti i livelli di conoscenza. Firenze. Testo disponibile sul sito: http:// www.acad.it/downloads/sillabo.html.

Arcangeli, M., Giugni, S., Cardillo, G., D’Angelo, K., Ferrari, S., Greco, N. \& Lucatorno, A. (2014). Attestato ADA. Piano dei corsi. Firenze: Alma \& Società Dante Alighieri.

Barni, M., Bandini, A., Sprugnoli, L., Lucarelli, S., Scaglioso, A. M., Strambi, B., Fusi, C. \& Arruffoli, A. M. (2009). Linee guida CILS. Certificazione di Italiano come Lingua Straniera (presentazione di M. Vedovelli). Perugia: Guerra Edizioni. Testo disponibile sul sito: https://goo.gl/toCDN9.

Benucci, A. (a cura di). (2007). Sillabo di Italiano per Stranieri. Una proposta del Centro Linguistico dell'Università per Stranieri di Siena. Perugia: Guerra.

Cardillo G. \& Vecchio P. (a cura di). (2015). Nuovo sillabo della Certificazione PLIDA. Livelli A1-C2. Roma: Società Dante Alighieri. Testo disponibile sul sito: https://goo.gl/bgGxxH (la parte integrale del NSP: Quaderni delle specifiche).

Lo Duca, M. G. (2006). Sillabo di italiano L2. Roma: Carocci.

Minciarelli, F. \& Comodi, A. (2005). Sillabo per i cinque gradi del corso di lingua e cultura italiana per stranieri. Perugia: Guerra.

Spinelli B. \& Parizzi F. (a cura di). (2010). Profilo della lingua italiana. Livelli di riferimento del QCER A1, A2, B1, B2. Firenze: La Nuova Italia.

Grammatiche didattiche della lingua italiana analizzate

Celi, M. \& La Cifra, L. (2011). Grammatica d'uso della lingua italiana. Teoria ed esercizi (A1/B2). Milano: Hoepli.

Chiuchiù, A. \& Chiuchiù, G. (2015). Comunicare in italiano. Grammatica per stranieri con esercizi e soluzioni (A1/C1). Milano: Hoepli.

Debetto, G. (2016). La lingua italiana e le sue regole (A1-B2). Torino: Loescher.

Ercolino, E. \& Pellegrino, T. A. (2011). L'utile e il dilettevole 1. Esercizi e regole per comunicare (A1/B1). Torino: Loescher.

Esposito, A. \& Errico, R. (2007). Grammatica di base. Risorse di grammatica italiana per stranieri (A1/B2). Perugia: Guerra. 
Gatti, F. \& Peyronel, S. (2006). Grammatica in contesto. Strutture e temi di italiano per stranieri (A1/B1). Torino: Loescher.

Iacovoni, G., Fiorentino, B. \& Persiani, N. (2009). Gramm.it. Grammatica italiana per stranieri con esercizi e testi autentici $(A 1 / C 1)$. Torino: Bonacci.

Landriani, M. R. (2012). Grammatica attiva. Italiano per stranieri (A1/B2+). Firenze: Le Monnier.

Latino, A. \& Muscolino, M. (2014). Una grammatica italiana per tutti 1. Regole d'uso, esercizi e chiavi per studenti stranieri (Vol. 1: livello elementare; A1/A2). Roma: Edilingua.

Mezzadri, M. (2003). Italiano essenziale. Testo di grammatica per studenti stranieri dal livello principianti (A1) al livello intermedio-alto (B2). Perugia: Guerra.

Mezzadri, M. (2016). Grammatica pratica della lingua italiana (A1/C1). Torino: Bonacci.

Nocchi, S. (2011). Nuova grammatica pratica della lingua italiana. Esercizi - test-giochi (A1/B2; edizione aggiornata). Firenze: Alma.

Petri, A., Laneri, M. \& Bernardoni, A. (2015). Grammatica di base dell'italiano. La prima grammatica cognitiva dell'italiano (A1-B1). Barcellona: Casa delle Lingue.

Ricci, M. (2011). Via della grammatica. Teoria, esercizi, test e materiale autentico per stranieri (A1/B2, elementare - intermedio). Roma: Edilingua.

Tartaglione, R. \& Benincasa, A. (2015). Grammatica della lingua italiana Per Stranieri. Regole · esercizi - letture · test (Vol. 1: di base; A1/A2). Firenze: Alma.

Altro

Berruto, G. (1987). Sociolinguistica dell'italiano contemporaneo. Roma: La Nuova Italia Scientifica.

Bertinetto, P. M., Burani C., Laudanna A., Marconi L., Ratti D., Rolando C. \& Thornton A. M. (2005). Corpus e Lessico di Frequenza dell'Italiano Scritto (CoLFIS). Testo disponibile sul sito: http://linguistica. sns.it/CoLFIS/Home.htm.

Bongi, M. (2008, 6 giugno). Uso dell'articolo e dell'aggettivo possessivo coi nomi di parentela. Consulenza linguistica dell'Accademia della Crusca. Testo disponibile sul sito: https://goo.gl/tUSTKD.

Chiuchiù, A., Fazi, M. C. \& Bagianti, M. R. (2007). I verbi italiani regolari e irregolari. Perugia: Guerra.

Colombo, A. \& Graffi, G. (2017). Capire la grammatica. Il contributo della linguistica. Roma: Carocci.

Dardano, M. \& Trifone, P. (1995). Grammatica italiana con nozioni di linguistica (terza edizione). Bologna: Zanichelli. 
Della Valle, V. \& Patota, G. (2015). Viva la grammatica (nuova edizione aggiornata). Milano: Sperling \& Kupfer.

De Mauro, T. (2000). Il dizionario della lingua italiana per il terzo millennio. Torino: Paravia. Il Nuovo De Mauro disponibile sul sito: https://dizionario.internazionale.it.

De Renzo, F. (2013). I verbi italiani. Coniugazione e regole d'uso dei verbi italiani più diffusi. Torino: Loescher.

Parodi, S. (2011). Dizionario. Verbi (Edizione aggiornata. Il verbo e le sue strutture generali, classificazione dei verbi, coniugazione dei verbi regolari e irregolari, $i$ verbi irregolari, composti e derivati). Santarcangelo di Romagna: RusconiLibri.

Sabatini, F. (1985). L"'italiano dell'uso medio": una realtà tra le varietà linguistiche italiane. In: G. Holtus \& E. Radtke (a cura di), Gesprochenes Italienisch in Geschichte und Gegenwart (pp. 154-184). Tübingen: G. Narr.

Salvi, G. \& Vanelli, L. (2004). Nuova grammatica italiana. Bologna: il Mulino.

Sensini, M. (1997). La grammatica della lingua italiana (in collaborazione con F. Roncoroni). Milano: Mondadori.

Słapek, D. (2016). Forme verbali alternative (regolari e irregolari) del passato remoto. Studia universitatis ereditati, 4 (1), 85-96. DOI: https://doi.org/10.26493/2350-54 43.4(1)85-96. Testo disponibile sul sito: http://www.hippocampus.si/issn/2350-5443.

Słapek, D. (2019). Sillabi (linee guida) per la lingua italiana non materna e la questione della grammatica. In F. Avolio, A. Nuzzaci \& L. Spetia (a cura di), Politiche e problematiche linguistiche nella formazione degli insegnanti (pp. 183-198). Lecce/Rovato: Pensa MultiMedia.

Treccani (s.d.). [quesito relativo a "mia mamma e la mia mamma". Lingua italiana (grammatica). Testo disponibile sul sito: https://goo. gl/nTyis6. 


\title{
THEORETICAL AND PRACTICAL DISCORDANCES IN THE PRESENTATION \\ OF CERTAIN RULES IN A1/A2 LEVEL ITALIAN GRAMMAR TEXTBOOKS FOR FOREIGN LANGUAGE LEARNERS
}

\begin{abstract}
Summary
In this article, we attempt to examine how various grammatical issues pertaining to the A1/A2 level of the Common European Framework of Reference (CEFR) are presented in Italian grammar books for foreign language learners, amongst which are the following: a) subject pronouns (lui, lei vs. egli, ella) and the plural courtesy form (voi vs. loro); b) the pronominal particle $c i$ with the verbs essere ( $c$ 'è, ci sono) and avere (as in ce l'ho); c) verbal inflection in irregular verbs in the Present tense and irregular short forms in the Imperative; d) the semantics of the verbs venire vs. andare and potere, sapere vs. riuscire; and e) possessive adjectives, particularly the use of the definite article with terms of kinship (mio fratello vs. il mio fratellino). Our analysis, carried out on 15 grammar textbooks for Italian language learning that make explicit reference to the CEFR and published by well-known publishers, show some weaknesses regarding the teaching of grammar, such as the total omission of a given problem, a certain selectivity (for example, a problem is not presented in its entirety), as well as various theoretical and practical disagreements amongst the books in the presentation of grammatical rules to foreign learners.

Keywords: Italian non-mother tongue, Italian grammar, Italian grammar for foreigners, teaching grammar, CEFR, teaching Italian as a foreign language.
\end{abstract}

